\title{
Effectiveness of health and fitness smartphone applications to improve dietary habits and physical activity in Omani adults
}

\author{
K. Al Zuhaibi, F. McCullough and A.M. Salter \\ University of Nottingham, Loughborough, Leicestershire, LE12 5RD, UK.
}

An obesogenic environment has been spreading across the word. Society is increasingly reliant on technologies for performing daily living tasks. Technology has been blamed as a contributor to unhealthy lifestyle behaviours, however it has also recently been being developed to play a role in health management and provide solutions to many limitations in traditional strategies. Web-based technologies, such as pedometers and smartphone apps have emerged recently as useful tools in the promotion of physical activity and weight management. Apps such as "Lose it" and "MapMyFitness" are now among the most popular apps in iOS and Android, and has been supporting millions of people globally.

In Oman, almost $92 \%$ of adults own a smartphone mobile and about $82 \%$ of the subscribers recently indicated that they had intermediate level of skills with mobile technologies ${ }^{(1)}$. The affluence of the Gulf region, has allowed ownership of such technological devices to be high. The prevalence of physical inactivity among Omani adults has been estimated to be $33 \%$ for men and $41 \%$ for women $^{(2)}$. For college students, the prevalence was $43 \%$ in men and $57.8 \%$ in women. Results from our recent survey study confirmed that a high proportion of the Omani population (particularly those over 30y of age) were either overweight or obese, and the majority of the study population were physically inactive ${ }^{(3)}$. The present study, therefore, was a pilot intervention study aimed to; investigate the effectiveness of smartphone apps on physical activity and dietary intake behaviours in Omani adults living in Nottingham. In order to test the effectiveness, data of the following parameters (weight, BMI, mean daily calorie intake, mean daily step counts, $\%$ of (carbohydrate, fat, protein) was collected and analysed.

Twenty one Omani adults (16 males and 5 females), who were active smartphone users, participated in the one month intervention study. Two smartphones apps (MapMyFitness and Lose it!) were used as study tools to improve health lifestyle behaviours. App data was sent individually to the investigator by email at the end of every week. The study was approved by the University of Nottingham Medical School Ethics Committee. Statistical analysis, using SPSS, included paired t-test, independent t-test and ANOVA.

\begin{tabular}{llll}
\hline Variables & Baseline phase & End of the study & Sig. \\
\hline Body weight & $74 \cdot 7 \pm 12 \cdot 2$ & $73 \cdot 6 \pm 12 \cdot 6$ & .023 \\
Body Mass Index (BMI) & $26 \cdot 3 \pm 3 \cdot 7$ & $26 \cdot 2 \pm 3 \cdot 6$ & .017 \\
Average daily energy intake & $1462 \cdot 1 \pm 638 \cdot 3$ & $1472 \cdot 2 \pm 617 \cdot 1$ & .919 \\
Average daily step count & $2490 \cdot 9 \pm 2556 \cdot 4$ & $6359 \cdot 6 \pm 4671 \cdot 5$ & .004 \\
\hline
\end{tabular}

No significant change was seen in either energy or individual macronutrient intake of the participants following the intervention. However, significant changes $(\mathrm{P}<0.05)$ were observed in the body weight and BMI and daily step count increased 2.5 -fold $(\mathrm{P}<0.01)$. These changes were not significantly influenced by baseline BMI category. Over $90 \%$ of study participants indicated they would recommend both apps to friends. To conclude, results from this study indicate that existing web-based technology, such as (smartphone apps), can be successfully used by Omani adults to improve lifestyle factors associated with the high prevalence of diet-related chronic disease in this population. Further research is required in this area to investigate the longer-term impact of using such apps.

K.A-Z was supported by a PhD studentship provided by the Omani government.

1. http://docplayer.net/8387825-Sultanate-of-oman-safety-on-the-line-exposing-the-myth-of-mobile-communication-security.html.

2. Mabry R, Owen N, Eakin E (2014) A national Strategy for Promoting Physical Activity in Oman-A call for action. Sultan Qaboos University Med $J$, vol. 14, Iss. 2, pp. e170-175.

3. Al Zuhaibi K, McCullough F, Salter AM (2015) Impact of dietary practices on metabolic health in Omani adults. Proceedings of the Nutrition Society 74, E343. 